\title{
The G protein-coupled oestrogen receptor, GPER1, mediates direct anti-inflammatory effects of oestrogens in human cholinergic neurones from the nucleus basalis of Meynert
}

\author{
Erica Sarchielli $^{1}$ | Giulia Guarnieri $^{1}$ | Eglantina Idrizaj $^{2}$ | Roberta Squecco $^{2}$ | \\ Tommaso Mello ${ }^{3}$ | Paolo Comeglio ${ }^{4}$ | Pasquale Gallina ${ }^{5}$ | Mario Maggi ${ }^{6}$ | \\ Gabriella B. Vannelli ${ }^{1}$ | Annamaria Morelli ${ }^{1}$ (D)
}

${ }^{1}$ Section of Human Anatomy and Histology, Department of Experimental and Clinical Medicine, University of Florence, Florence, Italy

${ }^{2}$ Section of Physiological Sciences, Department of Experimental and Clinical Medicine, University of Florence, Florence, Italy

${ }^{3}$ Clinical Gastroenterology Unit, Department of Experimental and Clinical Biomedical Sciences "Mario Serio", University of Florence, Florence, Italy

${ }^{4}$ Sexual Medicine and Andrology Unit, Department of Experimental and Clinical Biomedical Sciences "Mario Serio", University of Florence, Florence, Italy

${ }^{5}$ Division of Pharmacology and Toxicology, Department of Neuroscience, Psychology, Neurosurgery School of Tuscany, Drug Research and Child Health (NEUROFARBA), University of Florence, Florence, Italy

${ }^{6}$ Endocrinology Unit, Department of Experimental and Clinical Biomedical Sciences "Mario Serio", University of Florence, Florence, Italy

\section{Correspondence}

Annamaria Morelli, Department of Experimental and Clinical Medicine, University of Florence, Largo Brambilla 3, 50139 Florence, Italy.

Email:a.morelli@unifi.it

Funding information University of Florence; Ministry of Education, Universities and Research (MIUR), Italy, Grant/Award Number: FFABR2017; Ente Cassa di Risparmio Florence, Italy, Grant/Award Number: 2011.120

\begin{abstract}
It has been well established, particularly in animal models, that oestrogens exert neuroprotective effects in brain areas linked to cognitive processes. A key protective role could reside in the capacity of oestrogen to modulate the inflammatory response. However, the direct neuroprotective actions of oestrogens on neurones are complex and remain to be fully clarified. In the present study, we took advantage of a previously characterised primary culture of human cholinergic neurones (hfNBM) from the foetal nucleus basalis of Meynert, which is known to regulate hippocampal and neocortical learning and memory circuits, aiming to investigate the direct effects of oestrogens under inflammatory conditions. Exposure of cells to tumour necrosis factor (TNF) $\alpha$ (10 $\mathrm{ng} \mathrm{mL}^{-1}$ ) determined the activation of an inflammatory response, as demonstrated by nuclear factor-kappa B p65 nuclear translocation and cyclooxygenase-2 mRNA expression. These effects were inhibited by treatment with either $17 \beta$-oestradiol $\left(E_{2}\right)$ $\left(10 \mathrm{nmol} \mathrm{L} \mathrm{L}^{-1}\right)$ or $\mathrm{G} 1\left(100 \mathrm{nmol} \mathrm{L}^{-1}\right)$, the selective agonist of the $\mathrm{G}$ protein-coupled oestrogen receptor (GPER1). Interestingly, the GPER1 antagonist G15 abolished the effects of $E_{2}$ in TNF $\alpha$-treated cells, whereas the ER $\alpha / E R \beta$ inhibitor tamoxifen did not. Electrophysiological measurements in hfNBMs revealed a depolarising effect caused by $E_{2}$ that was specifically blocked by tamoxifen and not by G15. Conversely, G1 specifically hyperpolarised the cell membrane and also increased both inward and outward currents elicited by a depolarising stimulus, suggesting a modulatory action on hfNBM excitability by GPER1 activation. Interestingly, pretreating cells with TNF $\alpha$ completely blocked the effects of $\mathrm{G} 1$ on membrane properties and also significantly reduced GPER1 mRNA expression. In addition, we found a peculiar subcellular localisation of GPER1 to focal adhesion sites that implicates new possible mechanisms of action of GPER1 in the neuronal perception of mechanical stimuli. The results obtained in the present study indicate a modulatory functional role of GPER1 with respect to mediating the oestrogen neuroprotective effect against inflammation in brain cholinergic neurones and, accordingly, may help to identify protective strategies for preventing cognitive impairments.
\end{abstract}




\section{1 | INTRODUCTION}

During recent decades, accumulating evidence has shown that oestrogens are not only reproductive hormones, but also important modulators of various neuronal functions, in particular learning and memory. ${ }^{1}$ Beyond the hypothalamus, which operates the central regulation of the reproductive neuroendocrine axis, oestrogens have multiple targets in extrahypothalamic brain regions associated with cognitive processes, such as the cortex and hippocampus, both in the male and female brain. ${ }^{2}$ Several studies in animal models have demonstrated that the natural oestrogenic hormone $17 \beta$-oestradiol $\left(E_{2}\right)$ regulates hippocampal neurogenesis and structural plasticity, ${ }^{3,4}$ as well as hippocampal synaptic transmission. ${ }^{5}$ In addition to neurotrophic effects, multiple studies have also indicated that $E_{2}$ has potent neuroprotective actions ${ }^{6}$ and it has been suggested as a possible therapeutic approach for attenuating the consequences of cognitive ageing and the effects of brain injury, even preventing them. ${ }^{7}$ In clinical studies, it has been reported that the risk of developing Alzheimer's disease (AD), the most common form of dementia, is higher in women than in men, especially in post-menopausal women compared to age-matched men. ${ }^{8}$ However, controversy exists about the efficacy of oestrogen replacement therapy in preventing ADrelated cognitive impairment.

The precise mechanisms through which oestrogen signalling imparts its beneficial effects on brain structures are complex and not fully elucidated. The major contribution to our knowledge on oestrogen neuroprotective action comes from relevant studies in a variety of brain injury models in animals of both sexes, as reviewed recently. ${ }^{9}$ In particular, $\mathrm{E}_{2}$ treatment was proven not only to revert ovariectomised-induced cognitive deficits, ${ }^{10}$ but also to provide ischaemic neuroprotection improving behavioural recovery after experimental stroke in mice. ${ }^{11}$ Interestingly, a neuroprotective role has been established for locally synthesised $E_{2}$ in the brain as a result of the expression of the $E_{2}$ biosynthetic enzyme aromatase by neurones under normal conditions, ${ }^{12}$ as well as astrocytes following neural injury. ${ }^{13}$ Moreover, it has been proposed that, in the brain, $E_{2}$ acts in concert with neurotrophic factors, such as microglia-derived insulin growth factor 1 to synergistically promote cellular health in the context of the injured brain in experimental models. ${ }^{14} E_{2}$ was also able to enhance retrograde transport of the brain-derived neurotrophic factor, thus modulating forebrain circuits in rodents. ${ }^{15}$

One of the most investigated hypothesis is that the neuroprotective actions of $E_{2}$ are mediated, at least in part, by effects on cholinergic inputs to the hippocampus and cerebral cortex. ${ }^{16}$ Accordingly, the selective destruction of basal forebrain cholinergic neurones (BFCNs) blocks the beneficial effects of $E_{2}$ in the hippocampus. ${ }^{17,18}$ BFCNs comprise magnocellular neurones grouped in distinct cellular structures on the medial and ventral cerebral hemisphere, including the nucleus basalis of Meynert (NBM). These neurones provide the major cholinergic projections to the cerebral cortex, hippocampus and amygdala, thereby regulating crucial behavioural states, such as attention, memory and learning. ${ }^{19}$ Importantly, the degeneration and loss of NBM cholinergic neurones represents a pathological correlate of the well-documented cholinergic derangement in AD patients ${ }^{20}$ and is also predictive of cognitive impairments in Parkinson's disease patients. ${ }^{21}$ Several studies have provided evidence of $E_{2}$ mediated protective effects on BFCNs by increasing choline acetyltransferase (ChAT) expression, ${ }^{22}$ as well as an overal enhancement of cholinergic neurone function and survival. ${ }^{23}$

It is well documented that the central effects of $E_{2}$ are mediated by the classical oestrogen receptors (ERs), ER $\alpha$ and ER $\beta$, which function as transcription factors that are able to regulate the activity of different genes, as well as by the membrane-bound $\mathrm{G}$ protein-coupled oestrogen receptor GPER1 (also called GPR30), which mediates rapid non-genomic effects. ${ }^{24}$ Increasing evidence supports a major role for GPER1 with respect to mediating the neuroprotective effects of oestrogens on the main cognitively relevant brain structures. ${ }^{25}$ All types of oestrogen receptors have been localised in multiple brain areas and are expressed in varying densities in both sexes. ${ }^{26,27}$ However, it has been suggested that GPER1 activation may be particularly involved in mediating the positive effects of $E_{2}$ on cognitive performance at the level of cholinergic afferents innervating the hippocampus and cortex, ${ }^{28,29}$ thereby directly acting on hippocampal synaptic function. Moreover, it has been demonstrated that BFCNs express high levels of GPER1, ${ }^{28}$ and release acteyl choline (ACh) upon GPER1 activation, ${ }^{29}$ thus suggesting that GPER1 may also regulate hippocampal memory and cognitive function indirectly by influencing the activity of cholinergic inputs to the hippocampus.

A key protective role could reside in oestrogen capacity of modulating the inflammatory response. In the central nervous system, pro-inflammatory processes associated with ageing (ie, so-called 'inflamm-ageing') contribute to neurodegeneration and cognitive decline. ${ }^{30}$ Reducing circulating levels of oestrogen could therefore aggravate the neuroinflammatory response that normally occurs with ageing. ${ }^{31}$ Indeed, $\mathrm{E}_{2}$ controls neuroinflammation via a modulatory action on the activity of glial cells involved in the inflammatory response, as detected in animal models. ${ }^{32,33}$ However, it is unclear whether $E_{2}$ can have direct, anti-inflammatory effects on cholinergic neurones, in particular in the NBM. In this context, we recently demonstrated that a primary culture of cholinergic neurones isolated from the human foetal NBM (hfNBM cells) expresses all types of oestrogen receptors that may be differently involved in mediating oestrogenic action on growth, differentiation and maintenance of the cholinergic phenotype. ${ }^{34}$ In particular, GPER1 appeared to be crucially required for the oestrogenic action on increasing ChAT expression. ${ }^{34}$ Based on such evidence, we took advantage of the availability of this cellular system, which 
recapitulates a human neuronal behaviour, aiming to investigate the direct effects of $E_{2}$ under inflammatory conditions, as well as to better characterise GPER1 expression and function in neurones that are crucially involved as target of the neuroprotective effects of oestrogen.

\section{2 | MATERIALS AND METHODS}

\subsection{Cell culture}

All of the experiments were carried out using hfNBM primary cell cultures obtained and characterised as described previously. ${ }^{34}$ In brief, foetal brain biopsies (two female 12-week-old fetuses) were obtained from therapeutic medical abortions after women approved and signed an informed consent document, as reported previously. ${ }^{35}$ The brain area corresponding to the NBM was dissected under a stereomicroscope and incubated with $1 \mathrm{mg} \mathrm{mL} \mathrm{m}^{-1}$ collagenase type IV (Sigma-Aldrich Corp., St Louis, MO, USA). The cell suspensions were mechanically dispersed by pipetting and cultured in Coon's modified Ham's F12 medium (Euroclone, Milan, Italy) supplemented with 10\% foetal bovine serum (Hyclone, Logan, UT, USA). The NBM cholinergic identity of hfNBMs was demonstrated by the expression of the major components of the cholinergic system, including proteins important for ACh synthesis, transport and hydrolysis (ChAT, vesicular ACh transporter and $\mathrm{ACh}$ esterase, respectively), by the basal release of ACh by these cells in the culture medium, as well as by the expression of CALB1 along with both high- (TrkA) and low- (p75/CD271) affinity nerve growth factor receptors, as reported previously. ${ }^{34}$ Cells that had been frozen and stored in liquid nitrogen for 1-12 months survived well after thawing and showed the same cholinergic phenotype as it did before storing.

For the induction of inflammation, cells were treated with the cytokine tumour necrosis factor (TNF) $\alpha 10 \mathrm{ng} \mathrm{mL}^{-1}$ (Gibco, Gaithersburg, MD, USA) for 3 or 24 hours. For oestrogen stimulation, cells were cultured in serum/phenol red-free medium (Lonza, Allendale, NJ, USA) and treated with $\mathrm{E}_{2}\left(10 \mathrm{nmol} \mathrm{L} \mathrm{L}^{-1}\right.$; Sigma-Aldrich Corp.) or G1 (100 nmol L-1; Merck Millipore, Billerica, MA, USA) for 24 hours in presence or absence of the receptor inhibitors tamoxifen (TAM) (100 nmol L-1) or G15 (1 $\mu \mathrm{mol} \mathrm{L}{ }^{-1}$; Merck Millipore), administered 30 minutes before hormonal stimuli.

\section{2 | Real-time quantitative reverse transcription- polymerase chain reaction (qRT-PCR)}

Isolation of total RNA and cDNA synthesis were performed using the 'RNeasy Micro kit' (Qiagen, Valencia, CA, USA) and the iScript ${ }^{\mathrm{TM}}$ cDNA Synthesis Kit (Bio-Rad Laboratories, Hercules, CA, USA). The integrity of total RNA was assessed by electrophoresis on agarose gel stained with ethidium bromide. A real-time qRT-PCR was performed according to the fluorescent TaqMan methodology, as described previously. ${ }^{34}$
The expression of $18 \mathrm{~S}$ ribosomal RNA subunit was found to be the most stable and the optimal for data normalisation compared to other two widely used housekeeping genes, GAPDH and $\beta$-actin; therefore, it was chosen as reference gene and used for relative quantitation of the target genes. Primers and probes for the housekeeping and target genes were predeveloped assays (Life Technologies), as listed in Table 1. Data analysis was based on the comparative threshold cycle $\left(C_{t}\right)$ using the $2^{-\Delta \Delta C_{t}}$ method $^{36}$ and was carried out using the MylQ2 ${ }^{\text {TM }}$ Two-Color Real-Time PCR Detection System (Bio-Rad Laboratories).

\section{3 | MTT assay}

Cell viability was determined by MTT assay (Sigma-Aldrich Corp.) as described previously. ${ }^{37}$ Briefly, $8 \times 10^{3}$ cells were seeded in 96 -well plates in Coon's modified Ham's F12 medium supplemented with $10 \%$ foetal bovine serum. After 24 hours, cells were serum-starved for 8 hours and then treated with TNF $\alpha 10 \mathrm{ng} \mathrm{mL}^{-1}$ for a further 24 hours. Then, the medium was replaced and $10 \mu \mathrm{L}$ of MTT solution was added for 3 hours at $37^{\circ} \mathrm{C}$. The optical density was measured at $450 \mathrm{~nm}$ using a Multiskan FC spectrophotometer (Thermo Fisher Scientific, Waltham, MA, USA). Cell viability was expressed as relative percentage of viable cells over control, taken as $100 \%$ (mean \pm SEM), from three separate experiments performed in quadruplicate.

\section{4 | Flow cytometry}

As described previously, ${ }^{34}$ after fixation and permeabilisation, cells were resuspended in phosphate-buffered saline with $1 \%$ foetal bovine serum and incubated with anti-ChAT rabbit pAb (dilution 1:100; catalogue no. AB143; Merck Millipore) followed by incubation with Alexa Fluor immunoglobulin G secondary antibody $(\mathrm{H}+\mathrm{L}$; dilution 1:200; catalogue no. A11011; Molecular Probes, Carlsbad, CA, USA). Cells were analysed on a FACSCanto II instrument (BD Pharmingen, San Diego, CA, USA) using BD FACSDIVA (BD Biosciences, San Jose, CA, USA) and FLowso, version 10 (Tree Star Inc., Ashland, OR, USA).

TABLE 1 Identification number of predeveloped assays used for the quantitative reverse transcription-polymerase chain reaction

\begin{tabular}{|ll}
\hline Gene & Assay ID number \\
\hline Tumour necrosis factor $\alpha$ receptor 1 (TNFR1) & Hs00191821_m1 \\
\hline Tumour necrosis factor $\alpha$ receptor 2 (TNFR2) & Hs00609976_m1 \\
\hline Oestrogen receptor $\alpha(E R \alpha)$ & Hs01046818_m1 \\
\hline Oestrogen receptor $\beta$ (ER $\beta)$ & Hs01100358_m1 \\
\hline $\begin{array}{l}\text { G protein-coupled oestrogen receptor 1 } \\
\text { (GPER1/GPR30) }\end{array}$ & Hs00173506_m1 \\
\hline Cyclooxygenase 2 (COX2) & \\
\hline 18S ribosomal subunit & Hs00153133_m1 \\
\hline
\end{tabular}




\section{5 | Immunofluorescence}

Immunocytochemistry was performed as described previously ${ }^{34,38}$ using the primary antibodies: anti-ChAT rabbit pAb (dilution 1:200; catalogue no. AB143; Merck Millipore), anti-nuclear factor-kappa B (NF-kB) p65 (F-6) mouse mAb (dilution 1:100; catalogue no. sc8008; Santa Cruz Biotechnology, Santa Cruz, CA, USA), anti-GPER1 rabbit pAb (dilution 1:200; catalogue no. AER-050; Alomone Labs, Jerusalem, Israel) and anti-vinculin (hVIN-1) mouse mAb (dilution 1:200; catalogue no. V9131; Sigma-Aldrich Corp.), followed by Alexa Fluor-568 or 488 conjugated secondary antibodies (dilution 1:200; catalogue no. A11011 and A11029, respectively; Molecular Probes). Negative controls were performed avoiding primary antibodies. Slides were imaged with a Microphot-FXA microscope (Nikon, Tokyo, Japan) or with a SP2-AOBS confocal microscope (Leica, Wetzlar, Germany) through a $63 \times 1.4$ NA oil immersion objective (Leica) for double-stained slides. The number of cells with nuclear NF-kB p65 was calculated by counting 10 fields per slide from three different experiments. The coloc2 plugin of the extended IMAGEJ, version FIJI (https://imagej.net/Fiji) was used for vinculin/GPER1 co-localisation analysis; the Pearson's correlation coefficient (PCC) value above threshold was computed in six slices in the Z-axis for each image from three different experiments. PCC has a range of 1 (perfect correlation) to -1 (perfect but a negative correlation) with 0 denoting the absence of a relationship. ${ }^{39}$ The significance of colocalisation was tested according to Costes method, ${ }^{40}$ which considers $P>0.95$ to indicate a significant true co-localisation.

\section{6 | Electrophysiology}

The patch pipettes (3-7 M $\Omega$ ) for whole-cell current recordings were obtained using a vertical puller (Narishige, Tokyo, Japan) from borosilicate glass tubing (Harvard Apparatus, Cambridge, MA, USA) and were filled with a filling pipette solution containing $\left(\mathrm{mmol} \mathrm{L}^{-1}\right)$ : 130 $\mathrm{KCl}, 10 \mathrm{NaH}_{2} \mathrm{PO}_{4}, 0.2 \mathrm{CaCl}_{2}, 1 \mathrm{EGTA}, 5 \mathrm{MgATP}$ and 10 Hepes. The $\mathrm{pH}$ was set to 7.2 with $\mathrm{KOH}$. Coverslips with the adherent cells were superfused at a rate of $1.8 \mathrm{~mL} \mathrm{~min}^{-1}$ with physiological extracellular bath solution comprising $\left(\mathrm{mmol} \mathrm{L}^{-1}\right)$ : $150 \mathrm{NaCl}, 5 \mathrm{KCl}, 2.5 \mathrm{CaCl}_{2}$, $1 \mathrm{MgCl}_{2}, 10$ D-glucose and 10 Hepes (pH 7.4 with $\left.\mathrm{NaOH}\right)$. As reported previously, ${ }^{41}$ we obtained the whole-cell configuration after mild application of negative pressure. The access resistance ( $R a)$ was constantly checked during the experiments because only the cells with stable access resistance (changes < 10\%) were included in the analysis. The technique, set up and electronics are as previously described in details in Idrizaj et al ${ }^{41}$ and Squecco et al. ${ }^{42}$ Briefly, the patch pipette was connected to a micromanipulator and an Axopatch 200 B amplifier (Axon Instruments, Foster City, CA, USA). Current- and voltage-clamp protocol generation and data acquisition were controlled using an output and an input of the A/D-D/A interfaces (Digidata 1200; Axon Instruments) and PCLAMP, version 6 (Axon Instruments). Electrode capacitance was compensated before disrupting the patch. By switching to the current-clamp mode $(I=0)$ of the $200 \mathrm{~B}$ amplifier, we measured the resting membrane potential (RMP). The passive properties parameters were estimated in voltage clamp as reported previously. ${ }^{42,43}$ The cell linear capacitance $(\mathrm{Cm})$ was calculated by estimating the area beneath the capacitive transient and was used as an index of the cell surface area assuming that membrane-specific capacitance is constant at $1 \mu \mathrm{F} \mathrm{cm}{ }^{-2}$. Ion current activation was evoked from a holding potential (HP) of $-80 \mathrm{mV},-60$ or $-30 \mathrm{mV}$ by applying step voltage pulses, 1 second long, from -80 to $50 \mathrm{mV}$, in increments of $10 \mathrm{mV}$. The sampling time of the early part of the current traces (up to $50 \mathrm{~ms}$ ) was $50 \mu$ s to better observe the activation latency and the rising time of the fast ion currents. The sampling time was increased to $10 \mathrm{~ms}$, starting from $50 \mathrm{~ms}$ up to the end of the step. To eliminate capacitive and leak currents, we used the P4 procedure. The currents were low-pass filtered with a Bessel filter at $2 \mathrm{kHz}$. To allow proper comparison between the current evoked in cells of different dimensions, the amplitude value was normalised to $\mathrm{Cm}$, and the ratio $1 / \mathrm{Cm}$ is the current density. The occurrence of the different ionic currents evoked in our preparations was verified by means of the commonly used channel blockers: tetrodotoxin $\left(1 \mu \mathrm{mol} \mathrm{L}{ }^{-1}\right)$ for voltage-activated $\mathrm{Na}^{+}$currents, nifedipine for L-type $\mathrm{Ca}^{2+}$ current and tetraethylammonium hydroxide $(20 \mathrm{mmol} \mathrm{L}$ $\left.{ }^{1}\right)$ for delayed rectifier $\mathrm{K}^{+}$currents. All the drugs were prepared daily from stock solutions, just before use. The experiments were performed at room temperature $\left(22^{\circ} \mathrm{C}\right)$. The results of all the experiments are expressed as the mean \pm SEM.

\subsection{Statistical analysis}

Data were analysed statistically using an unpaired Student's $t$ test or one-way ANOVA followed by Tukey's or Bonferroni's post-hoc analysis for multiple comparisons. $P<0.05$ was considered statistically significant. The analysis was performed using SPSS, version 25.0 (IBM Corp., Armonk, NY, USA).

\section{3 | RESULTS}

\subsection{Effects of TNF $\alpha$ on hfNBMs}

To analyse whether hfNBMs could respond to inflammatory insults, we first verified the expression of TNF $\alpha$ receptors (Figure 1A). As detected by qRT-PCR, the mRNA expression of oestrogen receptors (ER $\alpha$, ER $\beta$, GPER1) (Figure 1A) was confirmed. ${ }^{34}$ In addition, we originally report that hfNBMs express both TNFR1 and TNFR2 isoforms of TNF $\alpha$ receptors, with the TNFR1 isoform being 5 log units more abundant than the TNFR2 one. The hfNBM cell phenotype was confirmed by immunocytochemistry, which showed a strong immunopositivity to ChAT (Figure 1B). Quantitative analysis via flow cytometry showed a high percentage of ChAT-positive cells $(95.28 \pm 4.57 \%)$ that remained unaffected by 24 hours of exposure to TNF $\alpha$ (10 $\mathrm{ng} \mathrm{mL}^{-1}$ ) in three independent experiments (94.67 $\pm 4.28 \% ; t=0.09, d f=4, P=0.93$ vs control) (Figure $1 C$ ). In 
(A)

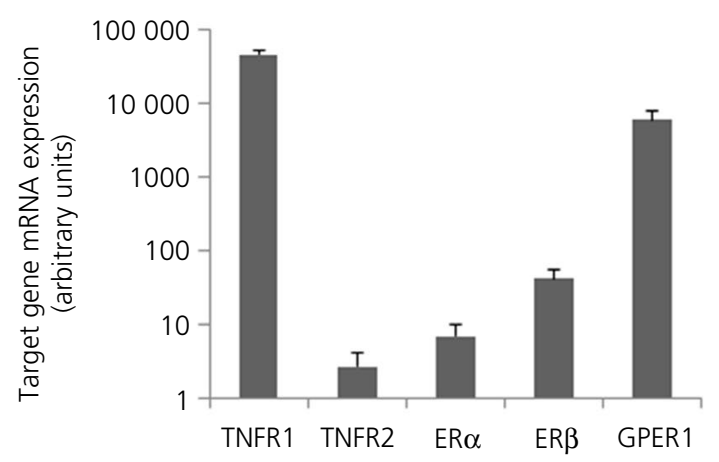

(B)

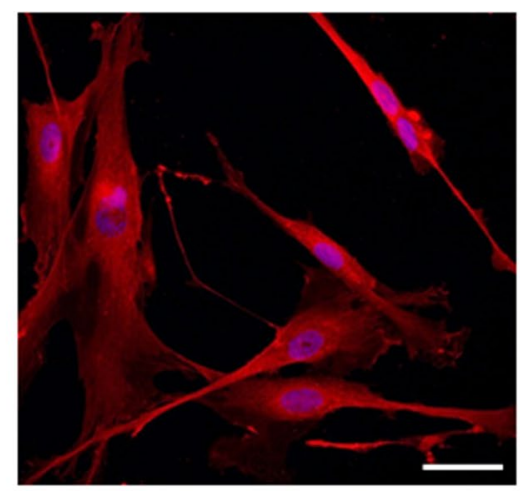

(c)

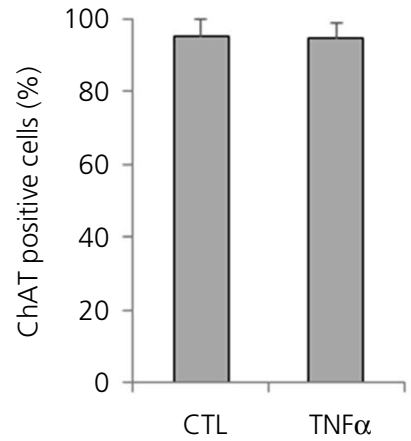

(D)

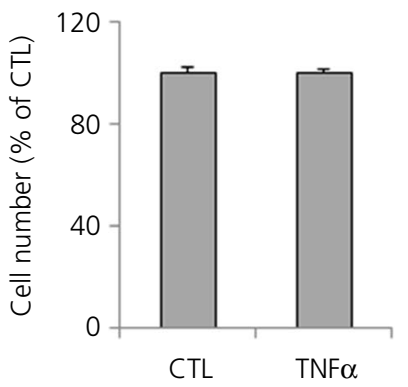

(E)

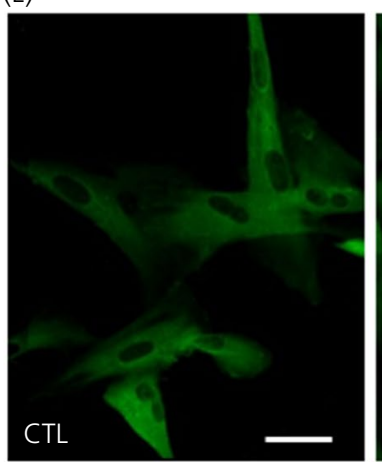

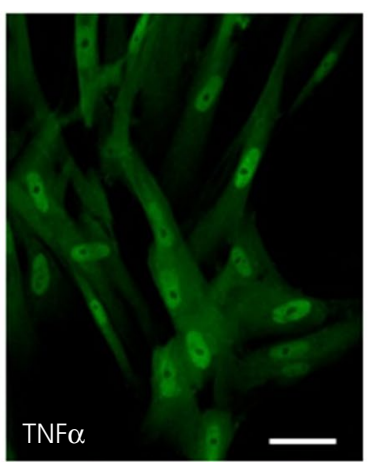

(F)

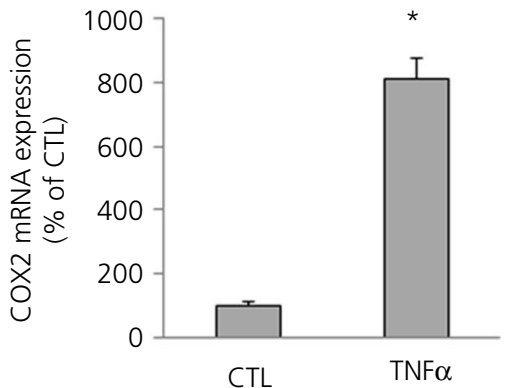

FIGURE 1 Tumour necrosis factor (TNF) $\alpha$ and oestrogen receptor (ER) expression and the effects of TNF $\alpha$ in hfNBMs. A, Relative mRNA expression by quantitative reverse transcription-polymerase chain reaction (qRT-PCR) analysis of TNFR1, TNFR2, ER $\alpha$, ER $\beta$ and G protein-coupled oestrogen receptor (GPER1) receptors normalised over the 18S ribosomal RNA subunit, taken as the reference gene, and reported as the mean \pm SEM $(n=6)$. B, Choline acetyltransferase (ChAT) expression in human cholinergic neurones (hfNBMs) as evaluated by immunofluorescence analysis (4',6-diamidino-2-phenylindole counterstained nuclei, scale bar $=50 \mu \mathrm{m}$ ). C, Flow cytometric analysis for ChAT; the bar graph shows the percentage of positive cells reported as the mean \pm SEM $(n=3)$. D, MTT analysis of hfNBMs treated or not (control [CTL]) with TNF $\alpha 10 \mathrm{ng} \mathrm{mL}^{-1}$ for $24 \mathrm{~h}(\mathrm{n}=3)$. E, Immunofluorescence analysis of nuclear factor-kappa B p65 nuclear translocation after TNF $\alpha$ stimulation (10 $\mathrm{ng} \mathrm{mL}^{-1}, 3 \mathrm{~h}$ ) compared to untreated cells (CTL) (scale bar $=50 \mu \mathrm{m}$ ). F, Cyclooxygenase 2 (COX2) mRNA expression in hfNBM cells by qRT-PCR after TNF $\alpha$ stimulation $\left(10 \mathrm{ng} \mathrm{mL}^{-1}, 24 \mathrm{~h}, \mathrm{n}=6\right.$ ). Data are normalised over the $18 \mathrm{~S}$ ribosomal RNA subunit and reported as percentage of CTL and displayed as the mean \pm SEM of three separate experiments performed in duplicate. ${ }^{*} P<0.001$ vs $C T L$, unpaired Student's $t$ test

addition, we determined whether TNF $\alpha$ treatment affected cell viability. The results shown in Figure 1D demonstrate that this is not the case $(t=0.05, d f=10, P=0.96)$. The inflammatory response of TNF $\alpha$ stimulated cells was investigated by subcellular immunolocalisation studies of NF-кB p65 (Figure 1E). In untreated cells, NF-кB p65 was totally retained within the cytoplasm (inactive), whereas exposure of cells to $10 \mathrm{ng} \mathrm{mL}^{-1}$ TNF $\alpha$ for 3 hours induced a full nuclear NF- $\mathrm{kB}$ p65 translocation (Figure 1E). Accordingly, a prolonged exposure to TNF $\alpha$ (24 hours) activated NF-кB p65 target gene transcription, resulting in an eight-fold increase of cyclooxygenase 2 (COX2) mRNA $(t=9.81, d f=10, P<0.001$ vs control) (Figure $1 \mathrm{~F})$.

\subsection{Effects of oestrogen on hfNBMs}

To determine whether oestrogens could interfere with the inflammatory response to TNF $\alpha$ in hfNBMs, serum-starved cells were exposed for 24 hours to either to $10 \mathrm{nmol} \mathrm{L^{-1 }} 17 \beta$-oestradiol $\left(E_{2}\right)$ or to the selective GPER1 agonist G1 (100 nmol L ${ }^{-1}$ ), or left untreated (control). Thereafter, culture medium was replaced and cells were stimulated with $10 \mathrm{ng} \mathrm{mL}^{-1} \mathrm{TNF} \alpha$ for 3 hours. As shown in Figure 2A, there were differences between the different treatments (one-way ANOVA, $F=153.39, d f=179, P<0.0001$ ) and, in particular, both $\mathrm{E}_{2}$ and $\mathrm{G} 1$ pre-treatments were able to significantly decrease the NF- $\mathrm{KB}$ p65 nuclear translocation induced by TNF $\alpha$ by almost $80 \%$ ( $P<0.001$, Tukey's post-hoc analysis) (Figure $2 \mathrm{~A}$ ). Interestingly, pre-incubating cells for 30 minutes with the $E R \alpha / E R \beta$ antagonist TAM (100 nmol L $\left.{ }^{-1}\right)$ did not prevent the $E_{2}$-mediated inhibition of NF-KB translocation, whereas the selective GPER1 antagonist $\mathrm{G} 15\left(1 \mu \mathrm{mol} \mathrm{L}{ }^{-1}\right)$ fully abolished the oestrogenic anti-inflammatory effect observed either with $E_{2}$ or with $G 1$ (Figure $2 A$ ). These results were confirmed by analysing the mRNA expression of the NF-kB target gene COX2 (one-way ANOVA, $F=78.06$, $d f=29, P<0.0001) . E_{2}$ treatment significantly reduced the TNF $\alpha$ induced increase in COX2 mRNA expression $(P<0.05$, Tukey's post-hoc analysis) (Figure 2B), whereas co-treatment with G15, 
(A)

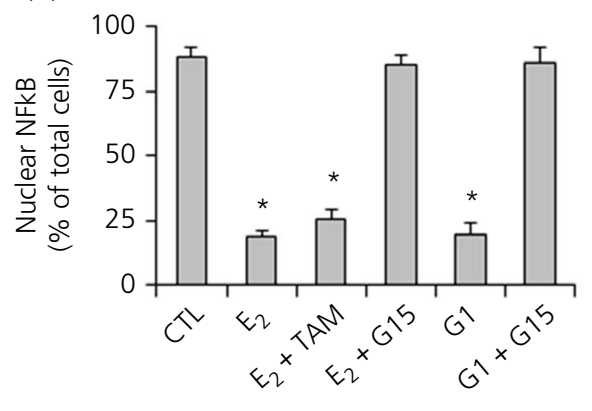

(B)

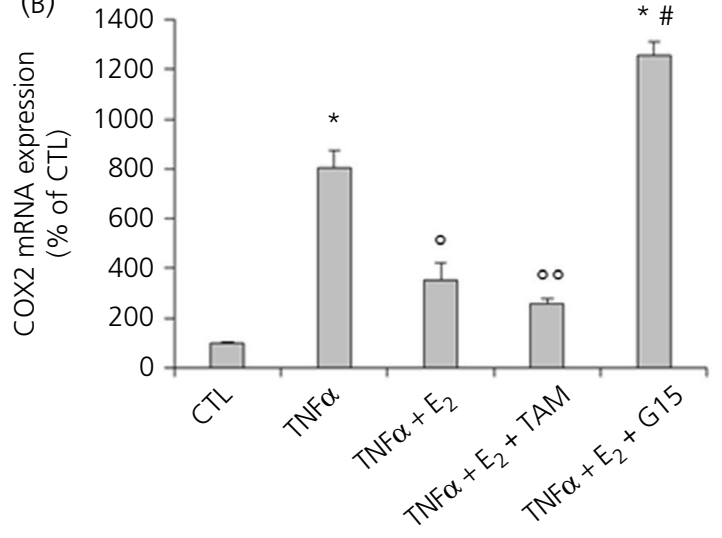

(c)

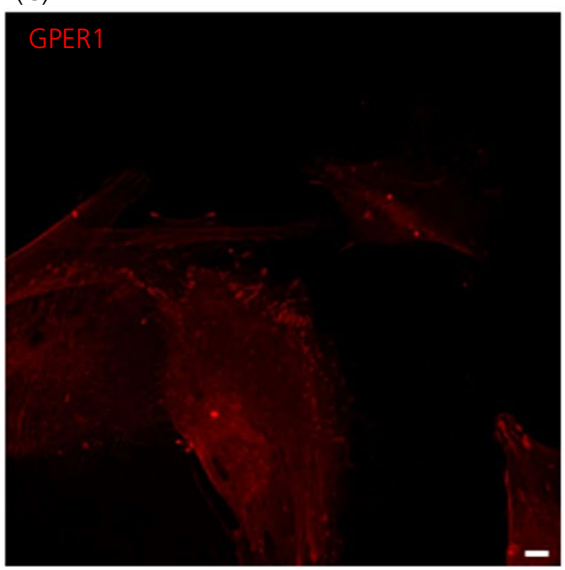

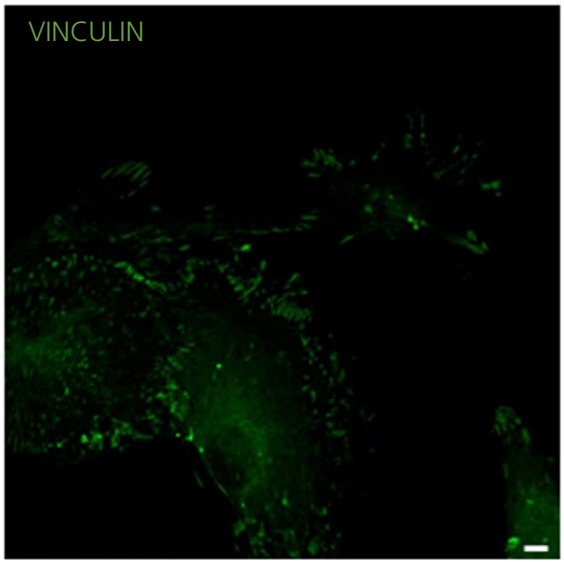

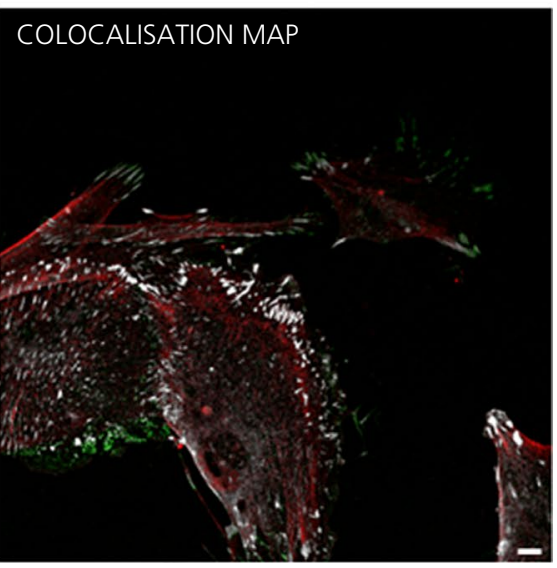

FIGURE 2 Oestrogenic effect on tumour necrosis factor (TNF) $\alpha$-induced inflammation pathway and G protein-coupled oestrogen receptor (GPER1) immunolocalisation analysis in human cholinergic neurones (hfNBMs). A, Quantification of nuclear factor-kappa B p65 nuclear translocation in TNF $\alpha$-stimulated $\left(10 \mathrm{ng} \mathrm{mL}^{-1}, 3 \mathrm{~h}\right)$ cells pretreated or not for $24 \mathrm{~h}$ with $17 \beta$-oestradiol $\left(\mathrm{E}_{2} ; 10 \mathrm{nmol} \mathrm{L}^{-1}\right)$ or G1 $\left(100 \mathrm{nmol} \mathrm{L}^{-1}\right)$ in the presence or not of tamoxifen $\left(100 \mathrm{nmol} \mathrm{L^{-1 }}\right)$ or $\mathrm{G} 15\left(1 \mu \mathrm{mol} \mathrm{L}^{-1}\right)$ and detected by immunocytochemistry. Data are expressed as percentage of cells with nuclear translocation/total cells (mean \pm SEM) calculated by counting 10 fields per slide of three separate experiments. ${ }^{*} P<0.001$ vs TNF $\alpha$ alone, indicated as control (CTL), one-way ANOVA followed by Tukey's post-hoc analysis. $\mathrm{B}$, Cyclooxygenase 2 (COX2) mRNA expression in TNF $\alpha\left(10 \mathrm{ng} \mathrm{mL}^{-1}\right)$ and $\mathrm{E}_{2}\left(10 \mathrm{nmol} \mathrm{L}^{-1}\right)$-stimulated cells (24 h) pretreated or not with Tamoxifen $\left(100 \mathrm{nmol} \mathrm{L}^{-1}\right)$ or G15 $\left(1 \mu \mathrm{mol} \mathrm{L}^{-1}\right)$ and analysed by quantitative reverse transcription-polymerase chain reaction (qRT-PCR). Data are normalised over $18 \mathrm{~S}$ ribosomal RNA subunit and reported as the percentage of CTL and displayed as the mean \pm SEM of three separate experiments performed in duplicate, ${ }^{*} P<0.001$ vs $C T L$, ${ }^{\circ} P<0.05$ and ${ }^{\circ}{ }^{\circ} P<0.01$ vs TNF $\alpha,{ }^{\#} P<0.001$ vs TNF $\alpha+E_{2}$; one-way ANOVA followed by Tukey's post-hoc analysis. C, Dual labelling immunofluorescence analysis of GPER1 (red) and the focal adhesion protein vinculin (green) in hfNBM cells. Co-localisation of GPER1 and vinculin is shown by white colour in the co-localisation map. Scale bar $=10 \mu \mathrm{m}$

but not TAM, significantly counteracted this effect $(P<0.001$ vs $T N F \alpha+E_{2}$ and vs CTL, Tukey's post-hoc analysis) (Figure 2B).

To further investigate GPER1 expression and activity in hfNBMs, we performed immunolocalisation and electrophysiological studies. Figure $2 \mathrm{C}$ shows the immunolocalisation of GPER1 in hfNBMs, as detected by confocal microscopy. Interestingly, double immunostaining with anti-GPER1 and anti-vinculin antibodies indicated a partial sublocalisation of GPER1 to focal adhesion sites (PCC $=0.30 \pm 0.03$; Costes $P$-value $=1$ ) (Figure $2 \mathrm{C}$ ).

Electrophysiological experiments were first carried out under current-clamp conditions to analyse the effects of G1 on RMP. The acute addition of $\mathrm{G} 1\left(100 \mathrm{nmol} \mathrm{L}^{-1}\right)$ to the external bath solution caused a rapid hyperpolarisation of the cell membrane compared to the control condition. A typical experiment is shown in Figure $3 \mathrm{~A}$, where the arrow next to the artefact indicates application of G1 to the bath solution. This hyperpolarising effect was completely prevented in cells treated with $\mathrm{G} 15\left(1 \mu \mathrm{mol} \mathrm{L} \mathrm{L}^{-1}\right)$ at least 30 minutes before addition of G1 (a representative record is shown in Figure $3 C$ ). The overall results from all of the experiments are shown in Figure 3B and were analysed with one-way ANOVA, indicating that the differences between the means were statistically significant ( $F=5.58 ; d f=31, P=0.009$ ). According to the Bonferroni's post-hoc analysis, we found that $\mathrm{G} 1$ induced a statistically significant membrane hyperpolarisation compared to CTL ( $P=0.013)$, which was abolished in the presence of G15 $(P=0.371)$. Cells treated with $\mathrm{G} 15+\mathrm{G} 1$ were significantly depolarised compared to those treated with $G 1$ alone $(P=0.014)$. We also tested the effect of $E_{2}\left(10 \mathrm{nmol} \mathrm{L}^{-1}\right)$ alone or in the presence of either TAM (100 nmol L-1) or G15 $\left(1 \mu \mathrm{mol} \mathrm{L}^{-1}\right)$ compared to CTL (Figure 3D). One-way ANOVA on the four independent 


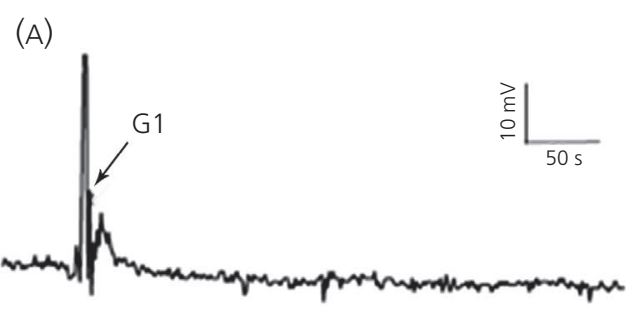

(C)

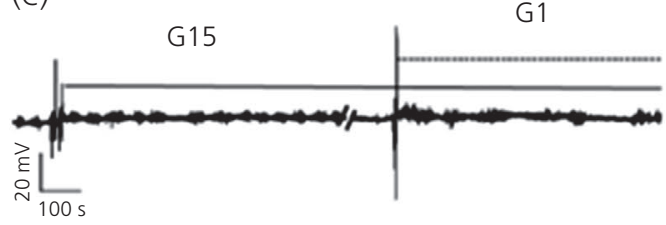

(E)

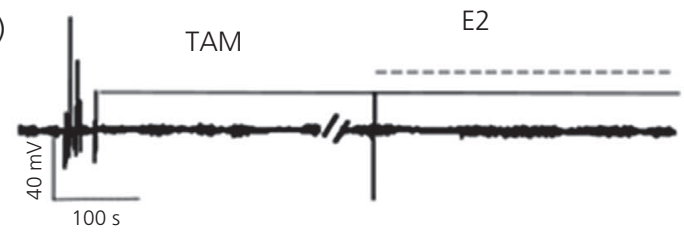

(F)

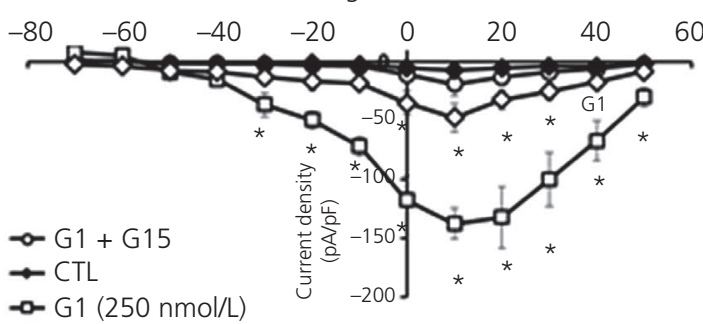

$\sim$ G1 (100 nmol/L)

(H)

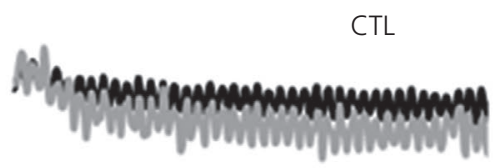

G1
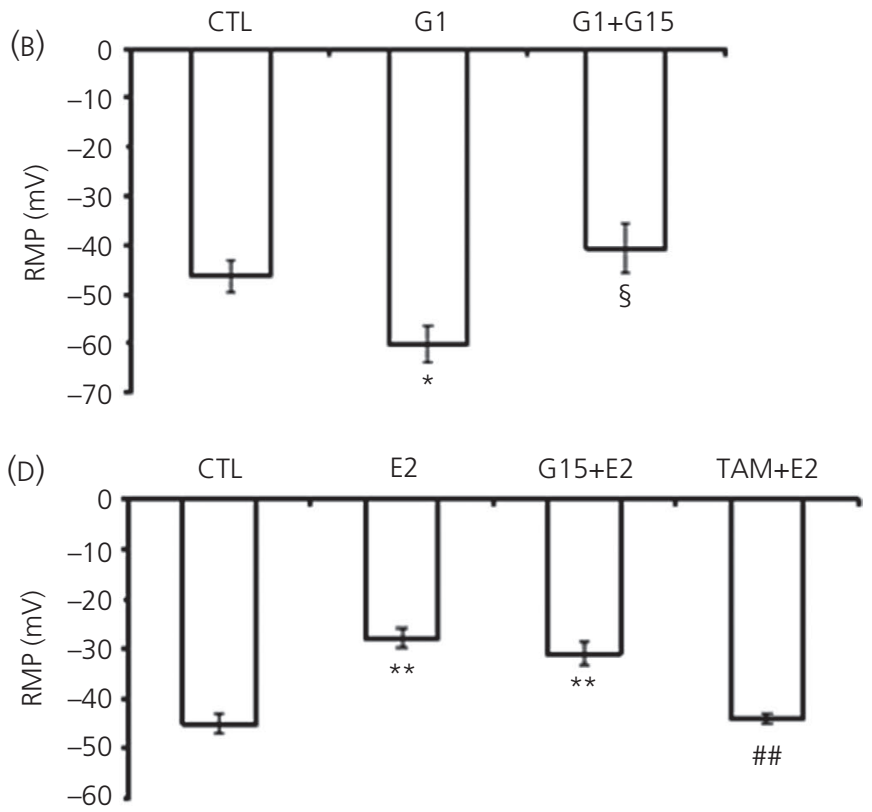

(G)

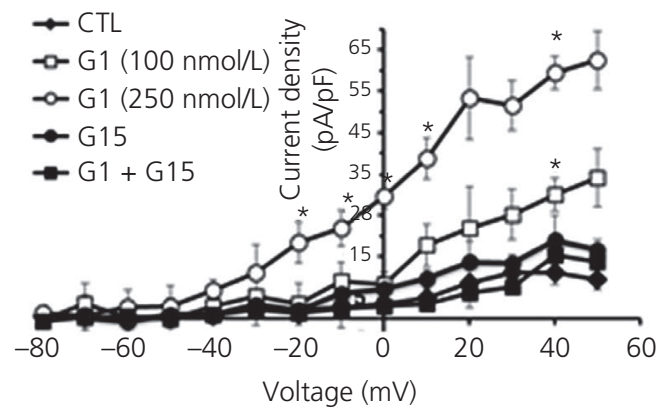

(I)

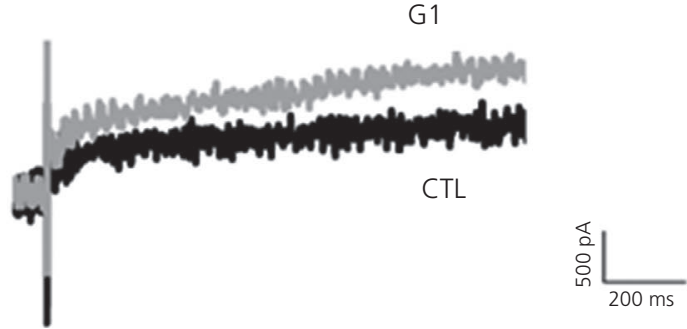

FIGURE 3 Effect of oestrogens on membrane potentials and ionic currents of human cholinergic neurones (hfNBMs). A, Representative resting membrane potential (RMP) tracing elicited in current clamp by $I=0 \mathrm{nA}$, from a cell after the acute addition of $\mathrm{G} 1\left(100 \mathrm{nmol} \mathrm{L}^{-1}\right.$ ) to the external bath solution (arrow next to the artefact). B, RMP values of hfNBMs under different conditions (mean values listed in Table 2): Control $(C T L)(n=14), G 1(n=13), G 1+G 15(n=5)$. Data are the mean \pm SEM. ${ }^{*} P<0.05$ vs $C T L,{ }^{\S} P<0.05$ vs $G 1$, one-way ANOVA followed by Bonferroni's post-hoc analysis. C, Typical tracing of RMP recorded in the presence of G15 (1 $\mu \mathrm{mol} \mathrm{L}^{-1}$; continuous line) and G1 (100 nmol L' ${ }^{1}$; dotted line). D, Summary data of the effects of $17 \beta$-oestradiol $\left(E_{2}\right)\left(10 n m o l L^{-1}, n=5\right), G 15+E_{2}(n=4)$ and tamoxifen $(T A M)+E_{2}(n=4)$ on RMP (mean values listed in Table 2). Data are the mean \pm SEM. ${ }^{* *} P<0.05$ vs CTL, ${ }^{\# \#} P<0.01$ vs $E_{2}$, one-way ANOVA followed by Bonferroni's post-hoc analysis. E, Representative tracing of RMP in the presence of TAM (100 nmol L-1; continuous line) applied 30 minutes before $\mathrm{E}_{2}\left(10 \mathrm{nmol} \mathrm{L} \mathrm{L}^{-1}\right.$; dashed line). $\mathrm{F}, \mathrm{I}-\mathrm{V}$ relationship of the inward ion currents recorded from hfNBMs in voltage clamp $(\mathrm{HP}=-80 \mathrm{mV})$ under control conditions (CTL, filled diamonds; $n=14)$, after the addition of $G 1$ (100 nmol L ${ }^{-1}$, open diamonds; $\left.n=13\right), G 1(250 \mathrm{nmol} / \mathrm{L}$, open squares; $\mathrm{n}=10$ ) or $\mathrm{G} 1+\mathrm{G} 15$ (open circles; $\mathrm{n}=12$ ) to the external bath solution. Data are the mean $\pm \mathrm{SEM}\left({ }^{*} P<0.05\right.$ vs $\mathrm{CTL}$ ). $\mathrm{G}, \mathrm{I}-\mathrm{V}$ plot of the outward ion currents recorded from hfNBMs in response to depolarising stimuli in control condition (CTL, filled diamonds; $n=6$ ), after the addition of $\mathrm{G} 1\left(100 \mathrm{nmol} \mathrm{L}^{-1}\right.$, open squares; $\left.\mathrm{n}=6\right)$, $\mathrm{G} 1\left(250 \mathrm{nmol} \mathrm{L} \mathrm{L}^{-1}\right.$, open circles; $\left.\mathrm{n}=3\right), \mathrm{G} 15\left(1 \mu \mathrm{mol} \mathrm{L} \mathrm{L}^{-1}\right.$; filled circles; $\left.\mathrm{n}=5\right)$ or $\mathrm{G} 1+\mathrm{G} 15$ (filled squares, $\mathrm{n}=5$ ) to the bath solution. Data are the mean \pm SEM. ${ }^{*} P<0.05$ vs CTL, one-way ANOVA followed by Bonferroni's post-hoc analysis. $\mathrm{H}$, Representative inward current traces resulting from test pulses to $+40 \mathrm{mV}$, from an hfNBMs cell under control conditions (CTL, black trace) and after the addition of $\mathrm{G} 1\left(100 \mathrm{nmol} \mathrm{L}^{-1}\right)$ (G1, grey trace). I, Representative outward current traces resulting from test pulses to $+40 \mathrm{mV}$, from an hfNBMs cell under control conditions (CTL, black trace) and after the addition of G1 (100 nmol L $\left.\mathrm{L}^{-1}\right)(\mathrm{G} 1$, grey trace) 
TABLE 2 Resting membrane potential (RMP) registered in human cholinergic neurones (hfNBMs) under different conditions

\begin{tabular}{lllllll} 
& CTL & G1 & G1 + G15 & $E_{2}$ & $E_{2}+T_{A M}$ & $E_{2}+G_{15}$ \\
RMP $(\mathrm{mV})$ & $-46.2 \pm 3.3$ & $-59.4 \pm 3.5^{*}$ & $-40.6 \pm 4.9^{\dagger}$ & $-27.9 \pm 2.0^{* *}$ & $-44.0 \pm 1.0^{\ddagger}$ & $-31.4 \pm 1.5^{* *}$ \\
\hline
\end{tabular}

${ }^{*} P<0.05,{ }^{* *} P<0.01$ vs control $(\mathrm{CTL}) ;{ }^{\dagger} P<0.05$ vs G1; ${ }^{\ddagger} P<0.01$ vs $17 \beta$-oestradiol $\left(\mathrm{E}_{2}\right)$, one-way ANOVA with Bonferroni's post-hoc analysis. TAm, tamoxifen.

populations showed statistically significant differences $(F=6.9$ $d f=26, P=0.0017)$. In this case, we observed that $E_{2}$ induced a statistically significant membrane depolarisation compared to CTL $(P<0.0001)$ (Figure 3D). This effect was completely prevented by TAM applied 30 minutes before $E_{2}\left(P<0.0001\right.$ vs $E_{2}$ alone; a representative experiment is shown in Figure $3 \mathrm{E})$, although not by G15 ( $P=0.207)$. All of the RMP values resulting from the above records are reported in Table 2.

To further characterise the GPER1-induced hyperpolarisation, we performed voltage-clamp experiments aimed at evaluating the ionic currents involved. Cells held at HP $=-80 \mathrm{mV}$ consistently showed inward ion currents elicited by a depolarising pulse protocol of stimulation. The acute addition of $\mathrm{G} 1\left(100 \mathrm{nmol} \mathrm{L}^{-1}\right)$ to the bath solution caused an increase in current amplitude that became even more evident using a higher concentration (250 nmol L-1) (Figure 4F). The statistical significance of the differences evaluated by one-way ANOVA ( $F=7.18, d f=350, P<0.00001$ ) between the different treatments at any voltage step is indicated by use of asterisks ( $P<0.05$; Bonferroni's post-hoc test) (Figure 3F). A typical inward current trace evoked by the $+40 \mathrm{mV}$ step pulse is shown in Figure $3 \mathrm{H}$, both in the control condition (CTL, black trace) and after the addition of $\mathrm{G} 1$ (100 nmol L-1) (G1, grey trace). A low percentage of cells (approximately 25\%) exhibited outward ion currents in response to the depolarising stimuli. These currents showed a small amplitude (CTL), although they were enhanced by G1 application, especially at the highest concentration used (Figure 3G). The statistical significance evaluated by one-way ANOVA $(F=20.57, d f=362$, $P<0.00001$ ) between the different treatments is indicated by the use of asterisks for any voltage step applied $(P<0.05$, Bonferroni's post-hoc test) (Figure 3G). Representative outward tracings evoked by the $+40 \mathrm{mV}$ step pulse are shown in Figure 3I, both in the control condition (CTL, black trace) and after the addition of G1 (100 nmol L' $\left.{ }^{1}\right)$ (G1, grey trace).

To investigate whether the inflammatory stimulus could interfere with the effects of GPER1 activation on both RMP and ion currents, we also performed the electrophysiological analysis on cells after 24 hours treatment with TNF $\alpha\left(10 \mathrm{ng} \mathrm{mL}^{-1}\right)$. One-way ANOVA on the four independent groups depicted in Figure 4A provided a statistical
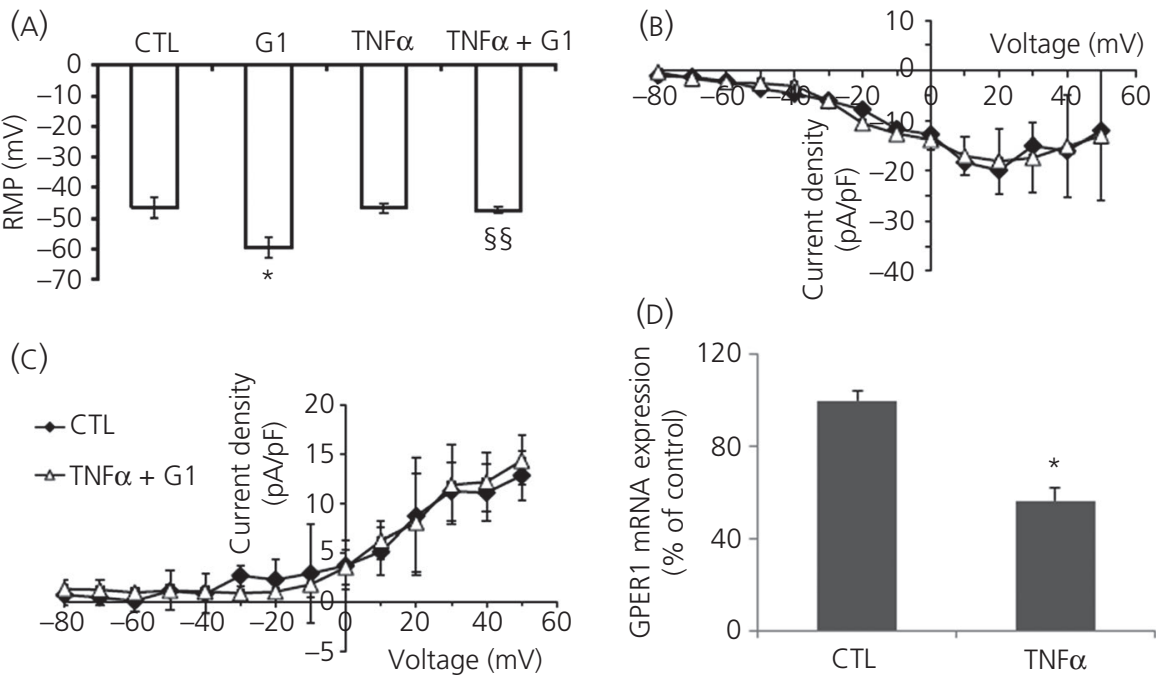

FIGURE 4 Effect of tumour necrosis factor (TNF) $\alpha$ on electrophysiological properties and G protein-coupled oestrogen receptor (GPER1) mRNA expression in human cholinergic neurones (hfNBMs). A, Summary data of the resting membrane potential (RMP) change recorded from hfNBMs following treatment with tumour necrosis factor (TNF) $\alpha\left(10 \mathrm{ng} \mathrm{mL}^{-1}\right.$ for $\left.24 \mathrm{~h} ; \mathrm{n}=12\right)$ or TNF $\alpha+\mathrm{G1}(\mathrm{n}=7)$. ${ }^{*} \mathrm{P}<0.05 \mathrm{vs}$ control (CTL), ${ }^{\S \S} P<0.01$ vs G1, one-way ANOVA followed by Bonferroni's post-hoc analysis. B, I-V relationship summarising the inward currents values recorded in TNF $\alpha$-treated cells after addition of $\mathrm{G} 1\left(100 \mathrm{nmol} \mathrm{L} \mathrm{L}^{-1}\right.$ ) (open up triangles, $\mathrm{n}=6$ ) and under control $(\mathrm{CTL}) \mathrm{conditions}(\mathrm{filled}$ diamonds, $\mathrm{n}=11)$. Current amplitudes did not turn out to be statistically different to $C T L(P>0.05$, unpaired Student's $t$ test). C, I-V plot summarising the outward currents values recorded under CTL conditions (filled diamonds, $n=6)$ and after G1 (100 nmol $\left.L^{-1}\right)$ addition in TNF $\alpha$-treated cells (open up triangles, $n=5)$. Current amplitudes did not turn out to be statistically different compared to $C T L(P>0.05$, unpaired Student's $t$ test). Data are the mean \pm SEM. D, Relative mRNA expression by quantitative reverse transcription-polymerase chain reaction analysis of GPER1 in hfNBMs treated or not $(C T L, n=6)$ with TNF $\alpha\left(10 \mathrm{ng} \mathrm{mL}^{-1}\right.$ for $\left.24 \mathrm{~h} ; \mathrm{n}=6\right)$; data were normalised over the $18 \mathrm{~S}$ ribosomal RNA subunit, taken as the reference gene, and reported as the mean \pm SEM. ${ }^{*} P<0.001$ vs CTL, unpaired Student's $t$ test 
significance $(F=4.31, d f=41, P=0.0103)$. With the post-hoc test, we found that TNF $\alpha$-treated hfNBMs showed a RMP similar to that observed in the CTL condition $(-46.6 \pm 1.6 \mathrm{vs}-46.2 \pm 3.3 \mathrm{mV})$ (Figure 4A). Acute addition of $\mathrm{G} 1$ did not cause significant changes in RMP when applied to TNF $\alpha$-treated cells $(-47.2 \pm 0.9 \mathrm{mV}$ ) (Figure 4A), whereas this RMP value was statistically different compared to that recorded when $\mathrm{G} 1$ was applied in untreated cells ( $P=0.0064$ vs G1) (Figure 4A).

We then evaluated the effect of G1 on the ion currents evoked on TNF $\alpha$-treated cells. When G1 was acutely added to the external bath solution, neither the inward, nor outward currents were found to be statistically different compared to those recorded in the control $(t=2.05, P=0.70$ for inward and $t=2.06, P=0.96$ for outward currents, respectively, Student's $t$ test) (Figure 4B,C). These results are consistent with the significantly reduced mRNA expression of GPER1 detected in hfNBMs treated with TNF $\alpha\left(10 \mathrm{ng} \mathrm{mL}^{-1}\right.$ for 24 hours) compared to untreated cells ( $t=5.69, d f=10, P<0.001)$ (Figure 4D), suggesting an inflammation-related impairment of GPER1 signalling.

\section{4 | DISCUSSION}

To date, knowledge of the mechanisms underlying the action of oestrogen in the brain has mainly derived from studies conducted in animal models, with very limited data being available concerning direct observations of the neurochemistry and physiology of human neurones. In the present study, we extended the characterisation of a human cellular model that recapitulates the typical features of NBM cholinergic neurones, ${ }^{34}$ further demonstrating their importance as a target of oestrogenic action in the human brain. Indeed, besides confirming that hfNBMs express classical ERs, our results indicate that the membrane-bound GPER1 is specifically implicated in protecting the functions of hfNBMs under inflammatory stimulus. Hence, our findings may help to clarify the mechanisms underlying the neuroprotective action of oestrogens on cognitive functions. Several experimental studies conducted in animal and in vitro models have demonstrated that the anti-inflammatory action of oestrogens is exerted via an inhibition of the inflammatory response by microglia and astroglia, reducing NF-kB p65 activity and the expression of pro-inflammatory cytokines. ${ }^{32,33,44}$ Recent findings suggested a novel $E_{2}$-mediated neuroprotective effect via regulation of microglia activation and promotion of the $\mathrm{M} 2$ anti-inflammatory phenotype in the brain of young adult ovariectomised rats. ${ }^{45}$ Moreover, there is evidence to suggest that neuroinflammation is capable of inducing glial aromatase expression in brain injury models, thus supporting the involvement of $E_{2}$ in modulating the brain response to proinflammatory mechanisms. ${ }^{32}$ However, less is known about direct, non-glia-mediated, anti-inflammatory effects of oestrogens on neurones. Therefore, a major finding of the present study is the demonstration, for the first time, that $\mathrm{E}_{2}$ may directly block inflammatory pathways in human NBM cholinergic neurones and that the GPER1 membrane receptor appears to be crucial with respect to mediating such action. Indeed, only the selective inhibition of GPER1 with G15 was capable of counteracting the inhibitory effect of $E_{2}$ on NF- $\mathrm{kB}$ p65 nuclear translocation, as well as the induction of COX2 mRNA determined by the TNF $\alpha$ stimulus, whereas tamoxifen, an antagonist of ER $\alpha$ and ER $\beta$ but not of GPER1, did not alter the oestrogenic effect. Although several studies suggest a role for both ER $\alpha$ and ER $\beta$ in mediating neuroprotective oestrogen actions, recent evidence has also demonstrated the crucial involvement of GPER1, for which selective activation was shown to perfectly mimic the oestrogenic effects by increasing the survival of murine cortical and hippocampal neurones exposed to neurotoxic insults. ${ }^{46}$ Moreover, in vivo studies conducted in the rat suggested that GPER1 activation may particularly contribute to the ability of $E_{2}$ to enhance cognitive performance by regulating the cholinergic afferents that innervate the hippocampus and cortex. ${ }^{28,29}$ Hence, it is conceivable that deficits in cholinergic function occurring with age and in menopausal women contribute to the loss of neuroprotective effect of oestrogens. We propose that the negative effects of 'inflamm-ageing' on cognitive and memory processes may be exacerbated by an oestrogenic decline that is responsible, at least in part, for a major vulnerability of NBM cholinergic neurones to the inflammatory insult, thus determining a cholinergic transmission deficiency. Indeed, we have also observed that the gene expression of GPER1 was significantly reduced in hfNBMs exposed to TNF $\alpha$. This finding suggests that the inflammatory stimulus may determine a reduced sensitivity of the receptor to its natural ligand $E_{2}$. Hence, only the high affinity binding with the selective agonist G1 can determine the protective effect mediated by GPER1 activation as observed in terms of blocking both NF-кB p65 nuclear translocation and downstream COX2 gene transcription. Electrophysiological analysis demonstrated that 24 hours of exposure to TNF $\alpha$ blocked the functional response of hfNBMs to $\mathrm{G} 1$, thus indicating the importance in preventing the deleterious effects of inflammation on mechanisms involved in the protective oestrogenic anti-inflammatory action. This is particularly relevant when considering pathological conditions, such as those linked to chronic neurodegenerative diseases, in which neuronal degeneration and death comprises a slow process accompanied by chronic neuroinflammation. In vivo studies have shown that $\mathrm{G} 1$ up-regulates the expression of both classical ERs and GPER in the hippocampus of aged female rats and counteracts ageing-related cognitive and behavioural alterations. ${ }^{47}$

Another important finding of the present study is the different electrophysiological response of hfNBMs to $E_{2}$ and $G 1$, when applied acutely. Our data suggest that excitability of hfNBMs may be regulated by oestrogens via differential mechanisms that depend on the recruited receptor. In particular, $E_{2}$ showed a depolarising effect that was specifically blocked by TAM and not by G15. Because membrane depolarisation is an essential function for neurone physiology, this effect could be related to oestrogen-dependent physiological functions natural occurring in hfNBMs and most likely responsible for an increased excitability and neurotransmitter release capacity. Conversely, G1 specifically hyperpolarised the cell membrane, thus suggesting that GPER1 activation could mediate 
inhibitory actions counteracting the depolarising response to $E_{2}$. Our findings are consistent with the differential control exerted by oestradiol on membrane properties as observed in gonadotrophin-secreting neurones. ${ }^{48}$ Chu et al ${ }^{48}$ showed that both stimulatory and inhibitory effects are elicited by acute oestradiol application and that the direction of response depended on dose and receptor subtype. In particular, in $\mathrm{GnRH}$ neurones, the oestradiol-induced depolarising effect was mediated by ER $\beta$ and was independent of GPER1 activation. Moreover, in hfNBMs in the present study, G1 was able to increase both inward and outward currents elicited by a depolarising stimulus, further suggesting a modulatory action on hfNBM excitability by GPER1 activation. In particular, we can hypothesise that, with respect to inducing outward and therefore hyperpolarising currents, even if only in a low percentage of cells (25\%), GPER1 activation may antagonise excitatory stimuli. In this regard, recent studies have demonstrated that, in different cellular systems, GPER1 and ER $\alpha$ can cross-talk with each other, highlighting the role of GPER1 as a modulator of classical oestrogen receptors. ${ }^{49}$ Indeed, GPER1 signalling appeared to be either synergic or antagonistic for the final output of oestrogenic, ER-mediated, action. ${ }^{50}$ Interestingly, $E R \alpha$ is reported to require GPER1 to mediate the neuroprotective actions of oestradiol on dopaminergic neurones in a mouse model of Parkinson's disease. ${ }^{51}$

Nonetheless, GPER1 can also act independently of ER $\alpha$ or ER $\beta$, as demonstrated in rat hippocampal neurones, where ER $\alpha$ and GPER1 activate different parallel signalling cascades. ${ }^{52,53}$ In particular, the neuroprotective action of selective oestrogen receptors modulators in rat neurones exposed to glucose-oxygen deprivation was dependent on GPER1 but independent of ER $\alpha$ or ER $\beta$ activation. ${ }^{54} \mathrm{~A}$ specific anti-inflammatory effect of GPER1 was also demonstrated in endothelial cells that was blocked by the concomitant activation of the classical ERs, indicating the opposite effects of the receptor subtypes. ${ }^{55}$ Interestingly, recent findings demonstrated that $\mathrm{E}_{2}$ potentiates hippocampal glutamatergic synaptic transmission similarly in both male and female rats, although distinct ER subtypes mediate the presynaptic and postsynaptic aspects of potentiation in each sex. ${ }^{56}$ Also, at the level of intracellular signalling, cAMP-activated protein kinase is required to initiate the $E_{2}$-induced potentiation only in females. ${ }^{57}$ L-type calcium channels and calcium release from internal stores are both required for $\mathrm{E}_{2}$-induced potentiation of hippocampal glutamatergic transmission in females, whereas, in males, either L-type calcium channel activation or calcium release from stores is sufficient to permit potentiation. ${ }^{57}$ Moreover, the $E_{2}$-induced increase of postsynaptic sensitivity to glutamate was mediated by GPER1 in female but not in male rats. ${ }^{56}$ Overall, these findings support the hypothesis of a neuromodulatory action of $E_{2}$ that could be exerted through the selective recruitment of its own receptors. Because hfNBMs have a female karyotype, it would be interesting, in further studies, to clarify the anti-inflammatory mechanisms of $E_{2}$ in neurones similar to hfNBMs with a male karyotype.

We acknowledge that the present study lacks a detailed characterisation clarifying the intrinsic mechanisms responsible for changes in membrane properties and also the nature of ionic currents elicited by GPER1 or classical ER activation in hfNBMs. However, we hypothesised that the hyperpolarising action of GPER1 activation could be ascribed to its known rapid effects on intracellular calcium mobilisation, ${ }^{24}$ which, in turn, could determine the consequent modulation of calcium-dependent ion channel opening. Accordingly, in mice, GPER1 activation increased the expression of the neuroprotective ion channel, small conductance calcium-activated potassium channel 2, improving neuronal survival following global cerebral ischaemia. ${ }^{58}$ Moreover, electrophysiological measurements in coronary artery smooth muscle cells have shown that GPER1 activation increased potassium efflux via large-conductance calcium-activated potassium channels. ${ }^{59}$

The very high abundance of GPER1 transcripts in hfNBMs, in comparison with the expression of classical ERs, further supports a crucial role of the receptor with respect to mediating the actions of oestrogen in this neuronal population. Indeed, immunolocalisation studies in the rat revealed that basal forebrain neurones that express GPER1 are mostly cholinergic (63\%-99\%). ${ }^{60}$ Interestingly, we also found a peculiar, although partial, localisation of GPER1 to focal adhesion vinculin-positive sites. Subsequent to the identification of GPER1, numerous studies have examined its subcellular localisation, which appeared to be unusual for a $\mathrm{G}$ protein-coupled receptor (GPCR), being localised to several sites within the cell. Depending on the cellular model, GPER1 was reported not only at the level of the plasma membrane, as expected for a GPCR, but also in the endoplasmic reticulum, Golgi apparatus and perinuclear space, thus suggesting intracellular actions and trafficking from intracellular compartments to the plasma membrane, as well as vice versa. Although further investigations are needed, based on our novel finding concerning a high level of GPER1/vinculin co-localisation, it is conceivable to hypothesise new possible mechanisms of action of GPER1 in neurones, which could be implicated in the perception of mechanical stimuli to regulate cell adhesivity, migration and synaptic plasticity, with all of these mechanisms being important for neuronal development and function. In this regard, recent studies have identified a family of adhesion GPCRs, comprising a large panel of 30 homologues within the GPCR superfamily displaying receptivity toward mechanical cues. ${ }^{61}$ Interestingly, vinculin is involved with anchoring actin to the membrane and plays a role in neurite extension and cell motility. ${ }^{62,63}$ Hence, the results obtained in the present study further suggest that GPER1 may mediate the effects of $E_{2}$ on neurite outgrowth in hfNBMs, as reported previously. ${ }^{34}$ Moreover, our findings appear to be in agreement with the demonstration that GPER1 stimulation with $E_{2}$ in different cellular systems (eg, breast cancer cells) promotes the formation of focal adhesions leading to the reorganisation of actin stress fibres and enhanced cellular adhesivity. ${ }^{64}$

Given the foetal origin of hfNBMs, we need to acknowledge that our findings may not exactly reflect the behaviour of neurones in response to neuroinflammation in the adult and ageing brain. However, obvious difficulties with respect to obtaining similar models from adult human NBM make our findings relevant, especially considering that hfNBMs showed molecular and 
functional features similar to those of mature NBM neurones in terms of the expression of cholinergic markers, receptor machinery, electrophysiological properties and the response to NGF, which is the main neurotrophin required for the survival and maintenance of this neuronal population. ${ }^{34}$

In conclusion, the results obtained in the present study indicate a functional role of GPER1 in human NBM cholinergic neurones, where it particularly appeared to be implicated in regulating the cell response to inflammatory stimuli and thereby mediating any direct neuroprotective actions in neurones. Together with previous studies demonstrating that, in hfNBMs, GPER1 not only is involved in mediating oestrogenic action on growth and differentiation, but also is necessary for maintaining the cholinergic phenotype, ${ }^{34}$ the present findings add new insights into the comprehension of oestrogenic action in the human brain. In particular, the neuroprotective mechanisms of action of GPER1 could be integrated in the signalling of the classical ERs. More detailed studies, including functional and mechanistic experiments, are needed to better clarify the position of GPER1 in the molecular cascade regulating human basal forebrain cholinergic neuron features in both healthy and pathological conditions, as well as in both sexes. This may help to optimally tune cell homeostasis and identify protective strategies for preventing neuronal damage associated with neurodegenerative diseases and cognitive disturbances.

\section{ACKNOWLEDGEMENTS}

The study was supported by the University of Florence (Fondi Ateneo Ricerca to AM), by FFABR2017 (funding for basic research activity) from the Ministry of Education, Universities and Research (MIUR), Italy to AM and by 'Ente Cassa di Risparmio Florence, Italy' (2011.120) to PG.

\section{CONFLICT OF INTERESTS}

The authors declare that they have no conflicts of interest.

\section{DATA AVAILABILITY}

The data that support the findings of the present study are available from the corresponding author upon reasonable request.

\section{ORCID}

Annamaria Morelli iD https://orcid.org/0000-0001-8027-9870

\section{REFERENCES}

1. McEwen BS, Akama KT, Spencer-Segal JL, Milner TA, Waters EM. Estrogen effects on the brain: actions beyond the hypothalamus via novel mechanisms. Behav Neurosci. 2012;126:4-16.

2. Gillies GE, McArthur S. Estrogen actions in the brain and the basis for differential action in men and women: a case for sex-specific medicines. Pharmacol Rev. 2010;62:155-198.

3. Mahmoud R, Wainwright SR, Galea LA. Sex hormones and adult hippocampal neurogenesis: regulation, implications, and potential mechanisms. Front Neuroendocrinol. 2016;41:129-152.

4. Sheppard PAS, Choleris E, Galea LAM. Structural plasticity of the hippocampus in response to estrogens in female rodents. Mol Brain. 2019;12:22.
5. Smejkalova T, Woolley CS. Estradiol acutely potentiates hippocampal excitatory synaptic transmission through a presynaptic mechanism. J Neurosci. 2010;30:16137-16148.

6. Arevalo MA, Azcoitia I, Garcia-Segura LM. The neuroprotective actions of oestradiol and oestrogen receptors. Nat Rev Neurosci. 2015;16:17-29

7. Engler-Chiurazzi EB, Brown CM, Povroznik JM, Simpkins JW. Estrogens as neuroprotectants: estrogenic actions in the context of cognitive aging and brain injury. Prog Neurogibol. 2017;157:188-211.

8. Snyder HM, Asthana S, Bain L, et al. Sex biology contributions to vulnerability to Alzheimer's disease: a think tank convened by the Women's Alzheimer's Research Initiative. Alzheimers Dement. 2016;12:1186-1196.

9. Azcoitia I, Barreto GE, Garcia-Segura LM. Molecular mechanisms and cellular events involved in the neuroprotective actions of estradiol. Analysis of sex differences. Front Neuroendocrinol. 2019:55:100787.

10. Engler-Chiurazzi EB, Singh M, Simpkins JW. From the 90's to now: a brief historical perspective on more than two decades of estrogen neuroprotection. Brain Res. 2016;1633:96-100.

11. Li J, Siegel M, Yuan M, et al. Estrogen enhances neurogenesis and behavioral recovery after stroke. J Cereb Blood Flow Metab. 2011;31:413-425.

12. Lu Y, Sareddy GR, Wang WX, et al. Neuron-derived estrogen regulates synaptic plasticity and memory. J Neurosci. 2019;39:2792-2809.

13. Peterson RS, Saldanha CJ, Schlinger BA. Rapid upregulation of aromatase mRNA and protein following neural injury in the zebra finch (Taeniopygia guttata). J Neuroendocrinol. 2001;13:317-323.

14. Sohrabji F, Williams M. Stroke neuroprotection: oestrogen and insulin-like growth factor-1 interactions and the role of microglia. J Neuroendocrinol. 2013;25:1173-1181.

15. Jezierski MK, Sohrabji F. Estrogen enhances retrograde transport of brain-derived neurotrophic factor in the rodent forebrain. Endocrinology. 2003;144:5022-5029.

16. Gibbs RB. Estrogen therapy and cognition: a review of the cholinergic hypothesis. Endocr Rev. 2010;31:224-253.

17. Gibbs RB, Wu D, Hersh LB, Pfaff DW. Effects of estrogen replacement on the relative levels of choline acetyltransferase, trkA, and nerve growth factor messenger RNAs in the basal forebrain and hippocampal formation of adult rats. Exp Neurol. 1994;129:70-80.

18. Lâm TT, Leranth C. Role of the medial septum diagonal band of Broca cholinergic neurons in oestrogen-induced spine synapse formation on hippocampal CA1 pyramidal cells of female rats. Eur J Neurosci. 2003:17:1997-2005.

19. Ferreira-Vieira TH, Guimaraes IM, Silva FR, Ribeiro FM. Alzheimer's disease: targeting the cholinergic system. Curr Neuropharmacol. 2016;14:101-115.

20. Kilimann I, Grothe M, Heinsen H, et al. Subregional basal forebrain atrophy in Alzheimer's disease: a multicenter study. J Alzheimers Dis. 2014;40:687-700.

21. Schulz J, Pagano G, Fernández Bonfante JA, Wilson H, Politis M. Nucleus basalis of Meynert degeneration precedes and predicts cognitive impairment in Parkinson's disease. Brain. 2018;141:1501-1516.

22. Gibbs RB, Pfaff DW. Effects of estrogen and fimbria/fornix transection on p75NGFR and ChAT expression in the medial septum and diagonal band of Broca. Exp Neurol. 1992;116:23-39.

23. Bora SH, Liu Z, Kecojevic A, Merchenthaler I, Koliatsos VE. Direct, complex effects of estrogens on basal forebrain cholinergic neurons. Exp Neurol. 2005;194:506-522.

24. Revankar CM, Cimino DF, Sklar LA, Arterburn JB, Prossnitz ER. A transmembrane intracellular estrogen receptor mediates rapid cell signaling. Science. 2005;307:1625-1630. 
25. Alexander A, Irving AJ, Harvey J. Emerging roles for the novel estrogen-sensing receptor GPER1 in the CNS. Neuropharmacology. 2017;113:652-660.

26. Mitra SW, Hoskin E, Yudkovitz J, et al. Immunolocalization of estrogen receptor beta in the mouse brain: comparison with estrogen receptor alpha. Endocrinology. 2003;144:2055-2067.

27. Hazell GGJ, Yao ST, Roper JA, Prossnitz ER, O'Carroll AM, Lolait SJ. Localisation of GPR30, a novel G protein-coupled oestrogen receptor, suggests multiple functions in the rodent brain and peripheral tissues. J Endocrinol. 2009;202:223-236.

28. Hammond R, Gibbs RB. GPR30 is positioned to mediate estrogen effects on basal forebrain cholinergic neurons and cognitive performance. Brain Res. 2011;1379:53-60.

29. Gibbs RB, Nelson D, Hammond R. Role of GPR30 in mediating estradiol effects on acetylcholine release in the hippocampus. Horm Behav. 2014;66:339-345.

30. Salvioli S, Capri M, Valensin S, et al. Inflamm-aging, cytokines and aging: state of the art, new hypotheses on the role of mitochondria and new perspectives from systems biology. Curr Pharm Des. 2006;12:3161-3171.

31. Brown CM, Mulcahey TA, Filipek NC, Wise PM. Production of proinflammatory cytokines and chemokines during neuroinflammation: novel roles for estrogen receptors alpha and beta. Endocrinology. 2010;151:4916-4925.

32. Pedersen AL, Brownrout JL, Saldanha CJ. Neuroinflammation and neurosteroidogenesis: reciprocal modulation during injury to the adult zebra finch brain. Physiol Behav. 2018;187:51-56.

33. Vegeto E, Belcredito S, Ghisletti S, Meda C, Etteri S, Maggi A. The endogenous estrogen status regulates microglia reactivity in animal models of neuroinflammation. Endocrinology. 2006;147:2263-2272.

34. Morelli A, Sarchielli E, Guarnieri G, et al. Young human cholinergic neurons respond to physiological regulators and improve cognitive symptoms in an animal model of Alzheimer's disease. Front Cell Neurosci. 2017;11:339.

35. Gallina P, Paganini M, Lombardini L, et al. Development of human striatal anlagen after transplantation in a patient with Huntington's disease. Exp Neurol. 2008;213:241-244.

36. Livak KJ, Schmittgen TD. Analysis of relative gene expression data using real-time quantitative $\mathrm{PCR}$ and the $2^{\text {(-Delta Delta } \mathrm{C}(\mathrm{T}))}$ method. Methods. 2001;25:402-408.

37. Ambrosini S, Sarchielli E, Comeglio P, et al. Fibroblast growth factor and endothelin-1 receptors mediate the response of human striatal precursor cells to hypoxia. Neuroscience. 2015;289:123-133.

38. Sarchielli E, Marini M, Ambrosini S, et al. Multifaceted roles of BDNF and FGF2 in human striatal primordium development. An in vitro study. Exp Neurol. 2014;257:130-147.

39. Adler J, Parmryd I. Quantifying colocalization by correlation: the Pearson correlation coefficient is superior to the Mander's overlap coefficient. Cytometry A. 2010;77:733-742.

40. Costes SV, Daelemans D, Cho EH, Dobbin Z, Pavlakis G, Lockett S. Automatic and quantitative measurement of protein-protein colocalization in live cells. Biophys J. 2004;86:3993-4003.

41. Idrizaj E, Sarchielli E, Morelli A, et al. Cortical and spinal conditioned media modify the inward ion currents and excitability and promote differentiation of human striatal primordium. J Chem Neuroanat. 2018;90:87-97.

42. Squecco R, Idrizaj E, Morelli A, Gallina P, Vannelli GB, Francini F. An electrophysiological study on the effects of BDNF and FGF2 on voltage dependent $\mathrm{Ca}(2+)$ currents in developing human striatal primordium. Mol Cell Neurosci. 2016;75:50-62.

43. Di Franco A, Guasti D, Squecco R, et al. Searching for classical brown fat in humans: development of a novel human fetal brown stem cell model. Stem Cells. 2016:34:1679-1691.
44. Giraud SN, Caron CM, Pham-Dinh D, Kitabgi P, Nicot AB. Estradiol inhibits ongoing autoimmune neuroinflammation and NFkappaBdependent CCL2 expression in reactive astrocytes. Proc Natl Acad Sci USA. 2010;107:8416-8421.

45. Thakkar R, Wang R, Wang J, Vadlamudi RK, Brann DW. 17ק-estradiol regulates microglia activation and polarization in the hippocampus following global cerebral ischemia. Oxid Med Cell Longev. 2018;2018:4248526.

46. Gingerich S, Kim GL, Chalmers JA, et al. Estrogen receptor $\alpha$ and G-protein coupled receptor 30 mediate the neuroprotective effects of $17 \beta$-estradiol in novel murine hippocampal cell models. Neuroscience. 2010;170:54-66

47. Wang J, Yu R, Han QQ, et al. G-1 exhibit antidepressant effect, increase of hippocampal ERs expression and improve hippocampal redox status in aged female rats. Behav Brain Res. 2018;359:845-852

48. Chu Z, Andrade J, Shupnik MA, Moenter SM. Differential regulation of gonadotropin-releasing hormone neuron activity and membrane properties by acutely applied estradiol: dependence on dose and estrogen receptor subtype. J Neurosci. 2009;29:5616-5627.

49. Hadjimarkou MM, Vasudevan N. GPER1/GPR30 in the brain: crosstalk with classical estrogen receptors and implications for behavior J Steroid Biochem Mol Biol. 2018;176:57-64.

50. Vajaria R, Vasudevan $\mathrm{N}$. Is the membrane estrogen receptor GPER1, a promiscuous receptor that modulates nuclear estrogen receptor-mediated functions in the brain? Horm Behav. 2018;104:165-172.

51. Bourque $M$, Morissette $M$, Di Paolo T. Neuroprotection in Parkinsonian treated mice via estrogen receptor $\alpha$ activation requires Gprotein-coupled estrogen receptor 1. Neuropharmacology. 2015;95:343-352.

52. Kim J, Szinte JS, Boulware MI, Frick KM. 17 $\beta$-Estradiol and agonism of G-protein-coupled estrogen receptor enhance hippocampal memory via different cell-signaling mechanisms. J Neurosci. 2016;36:3309-3321.

53. Briz V, Liu Y, Zhu G, Bi X, Baudry M. A novel form of synaptic plasticity in field CA3 of hippocampus requires GPER1 activation and BDNF release. J Cell Biol. 2015;210:1225-1237.

54. Abdelhamid R, Luo J, Vandevrede L, et al. Benzothiophene selective estrogen receptor modulators provide neuroprotection by a novel GPR30-dependent mechanism. ACS Chem Neurosci. 2011;2:256-268

55. Chakrabarti S, Davidge ST. G-protein coupled receptor 30 (GPR30): a novel regulator of endothelial inflammation. PLoS ONE. 2012;7:e52357.

56. Oberlander JG, Woolley CS. 17 $\beta$-Estradiol acutely potentiates glutamatergic synaptic transmission in the hippocampus through distinct mechanisms in males and females. J Neurosci. 2016;36:2677-2690.

57. Jain A, Huang GZ, Woolley CS, et al. GPER1/GPR30 activation improves neuronal survival following global cerebral ischemia induced by cardiac arrest in mice. Transl Stroke Res. 2012;3:500-507.

58. Kosaka Y, Quillinan N, Bond C, Traystman R, Hurn P, Herson P. GPER1/GPR30 activation improves neuronal survival following global cerebral ischemia induced by cardiac arrest in mice. Trans Stroke Res. 2012;3:500-507.

59. Yu X, Ma H, Barman SA, et al. Activation of $\mathrm{G}$ protein-coupled estrogen receptor induces endothelium-independent relaxation of coronary artery smooth muscle. Am J Physiol Endocrinol Metab. 2011;301:E882-E888.

60. Hammond R, Nelson D, Gibbs RB. GPR30 co-localizes with cholinergic neurons in the basal forebrain and enhances potassium-stimulated acetylcholine release in the hippocampus. Psychoneuroendocrinology. 2011;36:182-192. 
61. Scholz N, Monk KR, Kittel RJ, Langenhan T. Adhesion GPCRs as a putative class of metabotropic mechanosensors. Handb Exp Pharmacol. 2016;234:221-247.

62. Chen K, Zhang W, Chen J, Li S, Guo G. Rho-associated protein kinase modulates neurite extension by regulating microtubule remodeling and vinculin distribution. Neural Regen Res. 2013;8:3027-3035.

63. Miller KE, Suter DM. An integrated cytoskeletal model of neurite outgrowth. Front Cell Neurosci. 2018;12:447.

64. Magruder HT, Quinn JA, Schwartzbauer JE, Reichner J, Huang A, Filardo EJ. The G protein-coupled estrogen receptor-1, GPER-1, promotes fibrillogenesis via a Shc-dependent pathway resulting in anchorage-independent growth. Horm Cancer. 2014;5:390-404.
How to cite this article: Sarchielli E, Guarnieri G, Idrizaj E, et al. The G protein-coupled oestrogen receptor, GPER1, mediates direct anti-inflammatory effects of oestrogens in human cholinergic neurones from the nucleus basalis of Meynert. J Neuroendocrinol. 2020;32:e12837. https://doi. org/10.1111/jne.12837 